\title{
UN NUEVO MÉTODO DE DIAGNÓSTICO Y CUANTIFICACIÓN DE LA ANISEICONIA SUBJETIVA
}

\author{
A NEW METHOD TO QUANTIFY SUBJECTIVE ANISEIKONIA
}

\author{
GALINDO-FERREIRO A ${ }^{1}$, GALINDO-ALONSO J ${ }^{2}$, SÁNCHEZ-TOCINO H ${ }^{3}$
}

\begin{abstract}
RESUMEN
Objetivo: Diseñar un sistema de medida de la aniseiconia subjetiva así como validar éste en una muestra de sujetos para poderlo proponer para su uso en práctica clínica.

Material y métodos: Para la realización del estudio se utilizó un sinoptóforo Clement Clark 2051ltd y un par de test (uno para cada ojo del paciente) que se adaptan al portamiras. Las miras permiten la cuantificación de la diferencia de tamaño entre las imágenes subjetivas de los dos ojos, mediante una escala de proporcionalidad, en porcentaje de $0 \%$ a $30 \%$ con discriminación del 1\%. Se realizó un estudio observacional descriptivo transversal en 358 sujetos. Se les cuantificó la aniseiconia subjetiva con el nuevo método y se realizó una exploración oftalmológica completa. La muestra estaba compuesta por 3 grupos: emétropes, amétropes corregidos con gafas no progresivas y amétropes con lentes de contacto hidrofílicas.

Resultados: De los 358 sujetos un 32,7\% eran hombres y $67,3 \%$ mujeres con edad media de 38,34 (DE 17,5). Del total de la muestra un 40,2\% eran emétropes y 59,8\% amétropes. Al analizar el defecto de refracción valorado como equivalente esférico se detectó una predominancia de miopes respecto de hipermétropes. El grado de aniseiconia en el gru-
\end{abstract}

\begin{abstract}
Purpose: To design subjective aniseikonia measuring criteria in order to quantify its value and to use it to the clinical practice.

Material and Methods: In order to carry out the aniseikonia measurements, a sinoptophore (Clement Clark) was used and two tests (one per eye of each patient) were performed. A viewfinder capable of measuring the difference in size between subjective images in both eyes was used and a percentage scale of ratio between 0 and 30\%, with an accuracy of $1 \%$ was developed. An observational descriptive study was performed on 358 patients, and a complete ophthalmological examination also performed. The patients were divided in 3 groups: emmetropic patients, ametropic patients with non-progressive spectacles, and ametropic patients with hydrophilic contact lenses.

Results: Of the 358 patients studied, $22.7 \%$ were men and $67.3 \%$ women. The average age was 38.3 (SD 17.3) years. $40.2 \%$ of the patients were emmetropic and $59.8 \%$ had some type of ametropia. When analyzing the refractive defect measured as the spherical equivalent we found a higher prevalence of myopia than hyperopia. The level of aniseikonia in the group of emmetropic patients was $1.6 \%$ (SD 3.11), in patients with glasses was $1.3 \%$
\end{abstract}

Recibido: 21/4/06. Aceptado: 13/2/07

1 Doctor en Medicina. Servicio de Oftalmología. Complejo Hospitalario de Palencia.

2 Doctor en Física. Servicio de Oftalmología. Hospital Universitario Río Hortega.

3 Doctor en Medicina. Servicio de Oftalmología. Hospital Universitario Río Hortega.

Este estudio ha sido objetivo de la Tesis Doctoral de la Dra. Alicia Galindo Ferreiro.

Correspondencia:

Alicia Galindo Ferreiro

Clínica Galindo

C/. Francisco Zarandona, Esc derecha, 1. ${ }^{\circ} \mathrm{A}$

47003 Valladolid

España

E-mail: ali_galindo@yahoo.es 
po de emétropes fue de 1,6\%(DE 3,11), en los sujetos portadores de gafas fue de $1,3 \%$ (DE 2,81$)$ y en portadores de lentilla de $1,1 \%$ (DE 2,96). Tras comparar las medias de aniseiconia en pacientes anisométropes y no anisométropes no se detectaron diferencias estadísticamente significativas.

Conclusiones: Disponemos de un método sencillo, clínicamente útil para diagnosticar y cuantifificar la aniseiconia subjetiva.

Palabras clave: Aniseiconia subjetiva, sinoptóforo, cuantificación aniseiconia, anisometropía, imagen ocular.
(SD 2.81), and in patients with contact lenses was $1.1 \%$ (SD 2.96). Comparing the results of aniseikonia in anisometropic and emmetropic patients, no statistically significant differences were found.

Conclusion: A simple method to quantify subjective aniseikonia, useful in clinical diagnosis has been designed (Arch Soc Esp Oftalmol 2007; 82: 81-88).

Key words: Subjective aniseikonia, sinoptophore, aniseikonia measurement, anisometropia, ocular image.

\section{INTRODUCCIÓN}

La aniseiconia es una anomalía de la visión binocular por la cual cada ojo de un mismo paciente percibe la imagen de un mismo objeto con diferente tamaño $(1,2)$. Clasificaremos la aniseiconia dependiendo de la percepción del paciente como aniseiconia subjetiva/clínica y aniseiconia objetiva/teórica. La primera es la percepción que el sujeto refiere y la segunda es la que el explorador es capaz de determinar con métodos matemáticos.

La aniseiconia es uno de los mayores problemas que se presentan con ocasión de la prescripción de unas lentes correctoras cuando existe anisometropía. Si bien es cierto que desde el punto de vista teórico la aniseiconia está siendo objeto de estudio, en la práctica diaria de la oftalmología aún no se ha llegado a resolver el problema. La medición de esta patología no es una exploración habitual puesto que no se dispone en el mercado de un método para su cuantificación $(1,3)$, y por lo tanto no es posible tratarla correctamente.

El objetivo de nuestro estudio fue cuantificar la aniseiconia subjetiva, es decir, se diseño un método que permitiera medir la diferencia de tamaño aparente del objeto percibido en cada ojo por el paciente, para después aplicarlo a un grupo de sujetos y así validar el sistema de medida y proponerlo para su práctica clínica habitual.

\section{SUJETOS, MATERIAL Y MÉTODOS}

Se diseñó un test específico para adaptarlo al portamiras de un sinoptóforo Clement Clark 20511td.
Para ello se crearon dos objetos tales que la comparación de su tamaño por parte del paciente nos indicara el porcentaje de aniseiconia existente. Dado que los porcentajes buscados pueden ser del orden del $5 \%$ hemos creído que era difícil establecer comparaciones subjetivas directas entre dos objetos con tan escasa diferencia de tamaño, dificultad que se puede comprobar ensayando el test de Zeiss. Por ello se construyó una escala de proporcionalidad basada en lo siguiente.

\section{a) Fundamento de nuestro test}

Se suponen dos triángulos rectángulos que llamamos ABC y ADE de la figura 1a, donde se cumple que el segmento $\mathrm{DE}$ es proporcional a $\mathrm{BC}$, como el segmento $\mathrm{AE}$ lo es a $\mathrm{AC}$ (DE/BC $=\mathrm{AE} / \mathrm{AC})$. Por lo tanto, se puede calcular el tamaño de cualquier segmento vertical DE conociendo AC y la distancia $\mathrm{AE}$ al vértice $\mathrm{A}$, despejando nuestra incógnita $(\mathrm{DE}=\mathrm{AE} \cdot \mathrm{BC} / \mathrm{AC})$. La figura $1 \mathrm{~b}$ equivale al triángulo $\mathrm{ABC}$ de la figura $1 \mathrm{a}$, en el que se borra el cateto (segmento vertical) BC. La hipotenusa AB

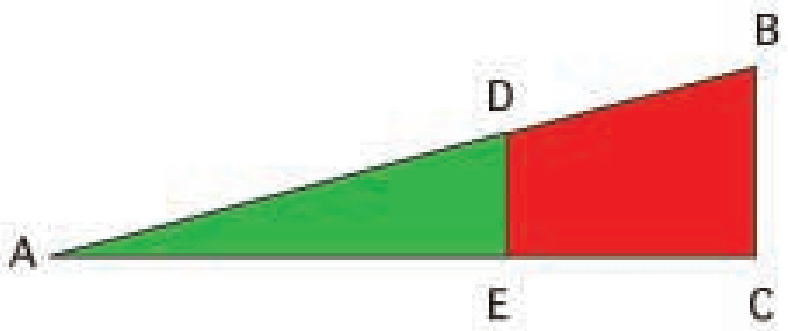

Fig. 1a: Triángulos equivalentes $A B C$ y $A D E$. 


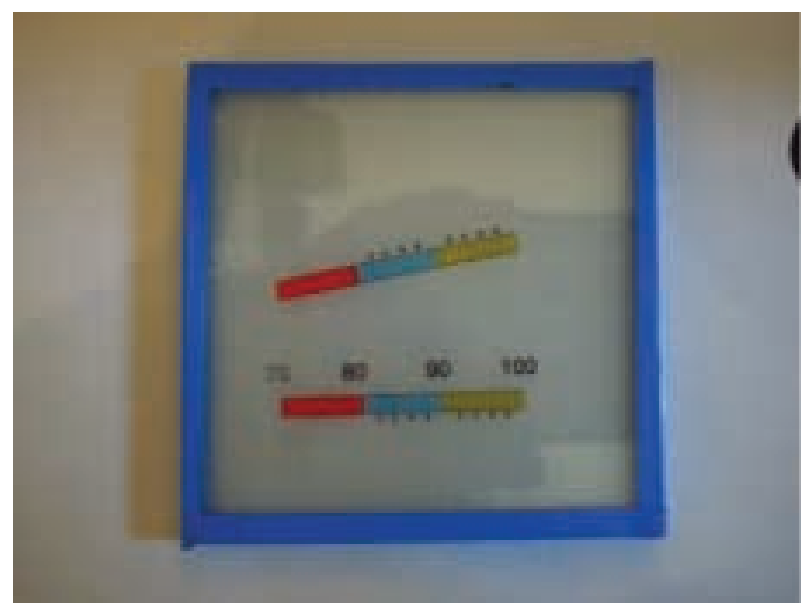

Fig. 1b: Mira con escala de proporcionalidad montada entre cristales del tamaño de las miras del sinoptóforo.

y el cateto $\mathrm{AC}$ se han dividido en diez segmentos para formar una escala, de modo que a cada porción de la escala se atribuye arbitrariamente el valor de longitud 10. El cateto BC se sustituye por una flecha del mismo tamaño (fig. 1c). Se supone que una flecha que encaja en las escalas en el valor 100 tiene asimismo una medida de 100 , considerado en sus propias unidades. Si se construyera otra flecha semejante a la de la figura 1c pero de un tamaño desconocido nos bastaría superponerla a la figura $1 \mathrm{~b}$ de forma que esté vertical y que sus extremos toquen ambas escalas. El valor de cualquiera de las escalas nos mediría la longitud de la flecha incógnita.

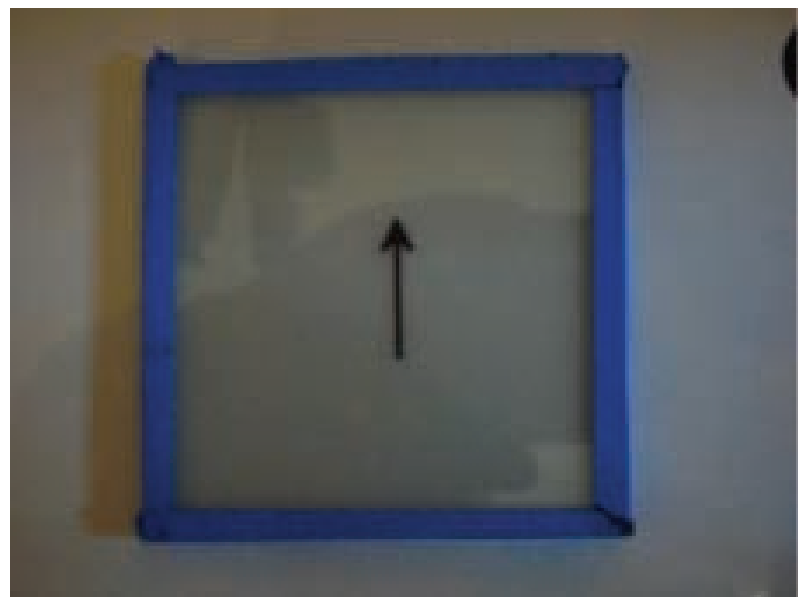

Fig. 1c: Flecha vertical que coincide con el tamaño del segmento vertical $B C$.

\section{b) El test}

Una vez diseñadas estas gráficas se decidió recortar el dibujo de la escala para evitar confusiones y centrar la atención del paciente en la porción de ésta que nos interesa, utilizando únicamente la zona comprendida entre el $70 \%$ y el $100 \%$, con un $1 \%$ de discriminación (fig. 1b). Estas gráficas se imprimieron (figs. 1 b y 1c) simultáneamente sobre una hoja transparente y luego se montaron entre cristales de la misma manera que los tests de serie del sinoptóforo. El tamaño de las figuras es igual al de los tests reglados de sinoptóforo de percepción simultánea, por ser los de mayor tamaño que admite el aparato y los más fáciles de ver.

Antes de colocar al paciente en el sinoptóforo, le explicamos adecuadamente la exploración a la que iba a ser sometido, totalmente inocua, rápida y simple. A través de los oculares el sujeto vería dos escalas y una flecha. En realidad, vería la escala con un ojo y la flecha con el otro.

\section{c) Hallar el porcentaje de aniseiconia}

Para hallar la diferencia porcentual de tamaño de imagen entre un ojo y otro bastaba con restar al $100 \%$ la cifra de la escala del test que el paciente nos comunicaba y se obtendría el tanto por cien de aniseiconia subjetiva que el paciente padecía. Para proceder siempre de la misma forma al realizar el estudio se colocó la flecha en el ojo derecho y la escala en el ojo izquierdo.

Se realizó un estudio observacional transversal descriptivo durante el periodo comprendido entre los años 2000 y 2003, para la cuantificación de la aniseiconia. Los criterios de exclusión fueron: la ausencia de visión binocular, el porte de cristales correctores progresivos o bifocales, el uso de lentes de contacto no hidrofílicas, la presencia de cualquier tipo de patología ocular anterior y posterior y cualquier discapacidad física o mental que no permitiera la exploración.

La muestra constó de 358 sujetos que se dividieron en tres grupos; portadores de gafas, los portadores de lentes de contacto y los pacientes emétropes. Existían pacientes que únicamente fueron valorados con gafas, o con lentillas sin embargo había un grupo de pacientes que eran portadores de gafas y lentillas y se valoró la aniseiconia subjetiva en ambas situaciones. Para posteriores com- 
paraciones se halló el equivalente esférico (EE) de los grupos y los pacientes fueron divididos en miopes cuando precisaban de corrección con lentes negativas $\leq 0,5$ dioptrías $(\mathrm{Dp})$, hipermétropes si llevaban lentes correctoras superiores a $+1 \mathrm{Dp}$ y emétropes cuando no precisaban corrección óptica. Además se definió anisometropía como la diferencia de graduación entre los ojos mayor o igual a $1 \mathrm{Dp}$.

A los efectos de este trabajo hemos de advertir que se consideró aniseiconia subjetiva cualquier cifra mayor del $0 \%$, pero se consideró patológico a las aniseiconias subjetivas mayores o iguales a $1 \%$.

\section{RESULTADOS}

De los 358 sujetos (716 ojos) analizados $32,7 \%$ fueron hombres y $67,3 \%$ mujeres. La edad media de la muestra fue de 38,34 (DE 17,5) años. Eran amétropes un 59,5\% (213 pacientes). De los sujetos amétropes un $39,25 \%$ (84 pacientes) eran portadores de lentillas. Si se tiene en cuenta el $n .^{\circ}$ de ojos, el defecto óptico de refracción más frecuente fue la miopía (37,8\% ojos derechos, y $37,6 \%$ ojos izquierdos del total de ojos amétropes). El porcentaje de anisometropia del total de los pacientes fue del $16,5 \%(\mathrm{~N}=59)$ con una media de anisometropía de 2,83 (DE 2,68) Dp.

Del total de los pacientes estudiados un $38,3 \%$ de los mismos tenían aniseiconia subjetiva. Al dividir la muestra de pacientes atendiendo a si éstos eran emétropes, amétropes portadores de gafas y amétropes portadores de lentillas la media de aniseiconia subjetiva y el porcentaje de sujetos con aniseiconia significativa $(>1 \%)$ fue:

- Grupo de emétropes: media 1,6 \%(DE 3,11), 60 pacientes $(35,7 \%)$ (fig. 2$)$.

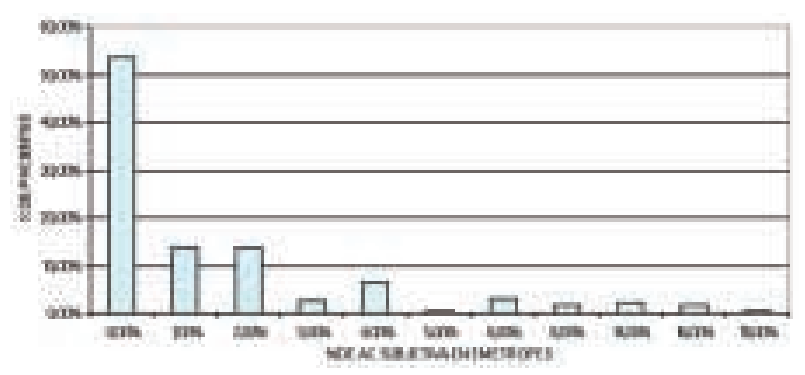

Fig. 2: Distribución de aniseiconia (AC) subjetiva en emétropes.
- Grupo de amétropes portadores de gafas $(\mathrm{N}=213)$ : media 1,3\% (DE 2,81), 76 pacientes (35,7\%) (fig. 3).

- Grupo de amétropes portadores de lentillas $(\mathrm{N}=84)$ : media 1,1\% (DE 2,96), 29 pacientes (30,95\%) (fig. 4).

No se encontraron diferencias significativas en la aniseiconia subjetiva por grupos de edad en pacientes portadores de lentillas y gafas, sin embargo si hubo diferencias significativas por grupos de edad en la aniseiconia subjetiva en el subgrupo de emétropes $(\mathrm{p}=0,002)$ que se localizaron en el grupo de edad mayor de 61 años (tabla I).

$\mathrm{Al}$ realizar una comparación para datos pareados bilateral $t$ student no se encontraron diferencias significativas entre aniseiconia subjetiva con gafas y lentillas $[\mathrm{N}=48, \mathrm{p}=0,537$, Media de diferencias $-0,25$, DE 2,78 y IC al $95 \%(-1,059,0,559)]$.

$\mathrm{Al}$ estudiar la media de aniseiconia subjetiva en ambos grupos amétropes atendiendo a si eran o no anisometropes no se detectaron diferencias significativas: así en portadores de gafas la media de ani-

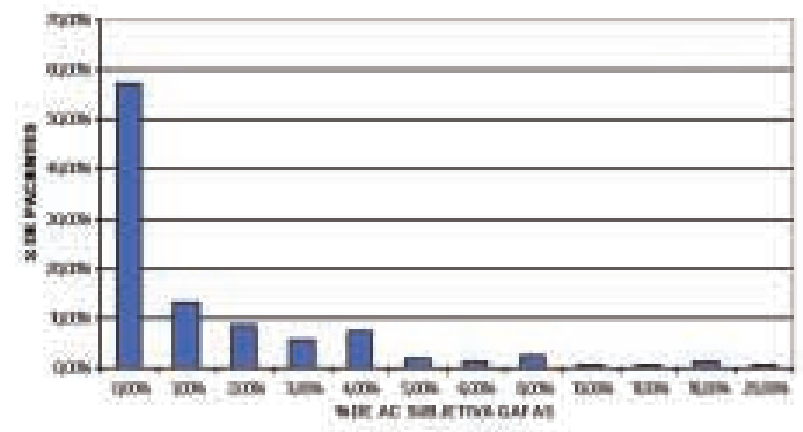

Fig. 3: Aniseiconia (AC) subjetiva en portadores de gafas.

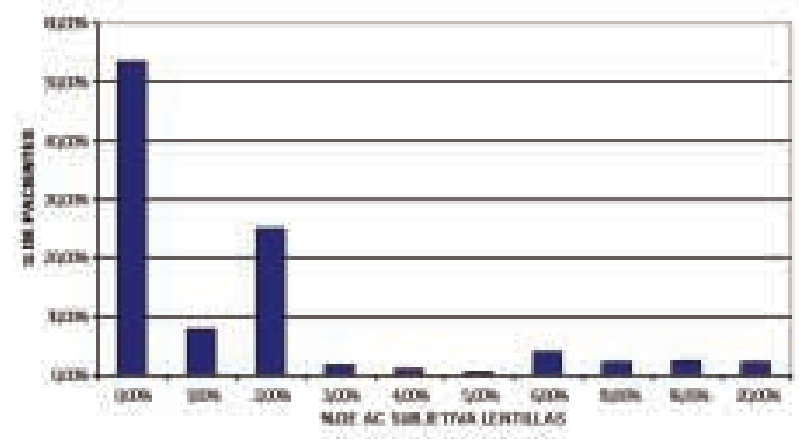

Fig. 4: Aniseiconia (AC) subjetiva en portadores de lentillas. 
seiconia subjetiva en anisométropes fue de 1,28\% (DE 2,98) y en no anisométropes de 1,26\% (DE $2,75)$ y en portadores de lentillas la media de aniseiconia subjetiva en anisométropes fue de 1,2\% (DE 3.54) y en no anisométropes de 1,09\% (DE 2,63).

El porcentaje de pacientes con aniseiconia subjetiva significativa en anisométropes y no anisométropes en el grupo de portadores de gafas fue del 43,9 y $41,6 \%$ respectivamente frente a 50,1 y $58,4 \%$ de pacientes sin aniseiconia en anisométropes y no anisométropes respectivamente. Estas diferencias no fueron estadísticamente significativas con una $\mathrm{p}=0,796$.

En el grupo de portadores de lentillas el porcentaje de pacientes con aniseiconia subjetiva en pacientes anisométropes fue de 53,3\% y en pacientes no anisométropes $51,4 \%$ frente a un porcentaje de 46,7 y $48,6 \%$ de anisométropes y no anisoméropes sin aniseiconia subjetiva. Estas diferencias tampoco fueron estadísticamente significativas con una $\mathrm{p}=0,897$ (tabla II).

\section{DISCUSIÓN}

En nuestro trabajo hemos realizado un screening de aniseiconia en 358 sujetos. Este número es menor que el estudiado por Hawkswell (4) en 1973 (1000 sujetos), Hughes (5) en 1937 (650 sujetos), pero mayor que las muestra de Burian (6) (320), Hicks (7) (280 sujetos), Linsk (8) (265 sujetos), Guillan (9) y Guillot (10) (100 sujetos).

Tabla I. Aniseiconia (AC0 subjetiva en pacientes emétropes por grupos de edad

\begin{tabular}{lcc}
\hline & $\begin{array}{c}\text { Media } \\
\text { Ac. subjetiva emétropes (\%) }\end{array}$ & DE \\
\hline $0-20$ años & 1,40 & 2,17 \\
21-40 años & 1,07 & 1,72 \\
41-60 años & 0,93 & 2,32 \\
$>60$ años & 3,53 & 5,41 \\
\hline \hline
\end{tabular}

Una vez revisada la bibliografía existente sobre los métodos de medida de la aniseiconia, resulta sorprendente que en estos últimos años no se disponga en el mercado de ningún instrumental adecuado para su estudio y cuantificación. Es una lástima que en 1970 se dejara de fabricar el eiconómetro espacial de la American Optical Corporation (11) que en nuestra opinión, ha sido el mejor método de medida de la aniseiconia subjetiva para uso en la clínica.

Wit (12) propone una nueva forma de medida de la aniseiconia, que el llama «Inspector de aniseiconia». En realidad, el método ya estaba descrito, pero él lo ha modernizado instalándolo en un programa de ordenador para Windows. Es el mismo diseño que el Nuevo Test de Aniseiconia de Awaya et al (NAT) (13). Sin duda nos parece un buen método, pero creemos que todavía no ha sido ensayado en un número apreciable de pacientes para declararlo útil, por lo que no podemos saber si verdaderamente funciona en la práctica diaria.

Podemos englobar nuestro método dentro del grupo de los métodos de prueba plana o amblioscopios, puesto que consiste en la comparación y cuantificación de la diferencia de tamaño de las imágenes percibidas de un objeto por los dos ojos. Weiss (14) y Winn (15) hicieron estudios de medición de aniseiconia subjetiva ideando unos tests que se adaptaban al sinoptóforo convencional, de forma parecida al método propuesto en este estudio. Estos autores refieren que el uso de un sinoptóforo adaptando unas figuras especiales tiene muchas ventajas para la medida de la aniseiconia subjetiva. Fontaine hace referencia al uso del sinoptóforo como método diagnóstico cualitativo de la aniseiconia subjetiva pero no llega a cuantificar ésta (16).

El diseño de nuestro test tiene la ventaja de que puede distribuirse como fichero informático, se puede imprimir en todo tipo de impresora de color y siempre será válido independientemente del tamaño exacto final. Además, es una exploración económica debido al bajo coste de los instrumentos necesarios. Consideramos que la interpretación de la

Tabla II. Porcentaje (\%) de pacientes con/sin aniseiconia (AC) subjetiva significativa atendiendo a anisometropía (ANISOM) o no anisometropía (NO ANISOM) en el grupo de pacientes portadores de gafas y lentillas

\begin{tabular}{lcccc}
\hline & ANISOM con AC & ANISOM sin & NO ANISOM con & NO ANISOM sin AC \\
\hline Gafas & $43,9 \%$ & $50,1 \%$ & $41,6 \%$ & $58,4 \%$ \\
Lentillas & $53,3 \%$ & $46,7 \%$ & $51,4 \%$ & $48,6 \%$ \\
\hline \hline
\end{tabular}


prueba por parte del explorador y el paciente es simple. Hemos comprobado que es un método reproducible, ya que durante la fase de desarrollo del análisis se repitió la medida al mismo paciente en varias ocasiones con un intervalo de tiempo y no se obtuvieron diferencias apreciables. Con el sinoptóforo podemos corregir defectos fisiológicos en la alineación de los ojos, puesto que los mandos y oculares del aparato son adaptables, como también observó Winn (15).

Como inconvenientes de nuestro método podemos mencionar la necesidad de un sinoptóforo, que no todo especialista posee. Es una exploración que se limita a un plano, puesto que únicamente determinamos la aniseiconia en el meridiano vertical. Recordemos que Winn (15) sólo cuantificaba la aniseiconia en un plano, escogiendo esta vez el meridiano horizontal. Como la mayor parte de los instrumentos usados para cuantificar la aniseiconia subjetiva descritos en la bibliografía, nuestro método tiene por objetivo la medición de la aniseiconia en mirada lejana.

Hemos encontrado que un $38,3 \%$ de los sujetos estudiados padecen aniseiconia subjetiva en porcentaje similar a los reportados por Hawkswell (4), Guillot (17), Burian (6) y Guillan (9) que encuentran una prevalencia del $42 \%$, y apreciablemente mayor al 10-15\% encontrado por Hughes (5).

En el grupo de los portadores de gafas encontramos aniseiconia subjetiva en el $35,7 \%$ de los pacientes, resultados similares a los descritos por Duke-Elder (18) (20-30\%). No hemos logrado encontrar referencias bibliográficas para establecer comparaciones con nuestros hallazgos ni de aniseiconia en emétropes ni en portadores de lentillas, aunque podemos afirmar que algunos autores revisados $(19,20)$ refieren haber encontrado paciente emétropes con aniseiconia subjetiva.

No se encontraron diferencias ni en la media ni en el porcentaje de pacientes con aniseiconia subjetiva entre anisométropes y no anisométropes tanto para portadores de gafas como portadores de lentillas. Ésta ausencia de diferencia podría deberse al escaso número de pacientes que se está comparando. Sin embargo a pesar que a priori existe un porcentaje alto de pacientes con aniseiconia subjetiva significativa, ninguno de estos pacientes refería sintomatología al respecto. La tolerancia de la aniseiconia varía de unas personas a otras, existiendo un umbral individual. Se define la aniseiconia clínica como la cantidad de aniseiconia que es preciso corregir para eliminar la sintomatología. La aniseiconia peor tolerada es la que crea una imagen con distorsión meridional y de forma acentuada si es oblicua. El sistema binocular puede compensar diferencias de hasta un 3\% de tamaños de imágenes retinianas, pero mayores diferencias podrían descompensar el sistema y entonces comenzaría a tener lugar la inhibición binocular.

Llama la atención que tanto la media de aniseiconia subjetiva como el porcentaje de pacientes que presentan ésta es muy similar entre pacientes portadores de gafas y lentillas. Esto está un poco en contradicción con lo que se cree entre los oftalmólogos acerca de que las lentillas disminuyen el grado de aniseiconia. En este estudio se tuvo en cuenta el modo de corrección de la ametropía (porte de gafas o de lentillas) porque sabemos que estos factores ópticos influyen en el tamaño de la imagen retiniana (aniseiconia objetiva) y por lo tanto, pensamos en principio que podrían influir en la percepción que el sujeto tiene de ésta (aniseiconia subjetiva); como se ha mostrado en los resultados, sin embargo no se encontraron diferencias estadísticamente significativas entre las medias de aniseiconia subjetiva al comparar portadores de gafas y lentillas.

Como conclusión se podría afirmar que existe un porcentaje de sujetos importante con aniseiconia subjetiva en la población estudiada aunque no todos sufren de clínica sugerente de aniseiconia. La sintomatología que normalmente refieren no es característica de aniseiconia sino que corresponde a una astenopía de fijación (molestias de la visión binocular, cefalea, sensación nauseosa crónica, dificultad de fusión de las imágenes, diferente tamaño del objeto al compararlo entre un ojo y otro..). Además sabemos que la tolerancia de la aniseiconia varía de unas personas a otras, existiendo un umbral individual (17). Se define la aniseiconia clínica como la cantidad de aniseiconia que es preciso corregir para eliminar la sintomatología. Normalmente ésta ocurre cuando la diferencia de tamaño percibida entre los dos ojos se acerca a 0,75\% (21). La aniseiconia peor tolerada es la que crea una imagen con distorsión meridional y de forma acentuada si es oblicua. El sistema binocular puede compensar diferencias de hasta un 3\% de tamaños de imágenes retinianas, pero mayores diferencias podrían descompensar el sistema y entonces comenzaría a tener lugar la inhibición binocular (11).

El disponer de un método de cuantificación de la aniseiconia subjetiva podría ser el primer paso para 
intentar explicar esta clínica y posteriormente buscar un modo de solucionarla.

\section{BIBLIOGRAFÍA}

1. Aguilar M, Mateos F. Óptica Fisiológica. Tomo 2. Valencia: Servicio de Publicaciones. 1993.

2. Berens C, Bannon RE. Aniseikonia. A present appraisal and some practical considerations. Arch Ophthalmol 1963; 70: 181-188.

3. Sampson WG. Applied optical principles. Int Ophthalmol Clin 1971; 11: 81-102.

4. Hawkswell A. Routine aniseikonic screening. Br J Physiol Opt 1974; 29: 126-129.

5. Hughes WL. Aniseikonia in emetropia. Am J Opthal 1937; 20: $887-890$.

6. Burian HM, Walsh R, Bannon E. Note on the incidence of clinically significant aniseikonia. Am J Ophthalmol 1946; 29: 201-203.

7. Hicks AM. A review of 200 consecutive cases examined on the eikonometer. Arch Ophthalmol 1943; 30: 298-311.

8. Linksz A. The diagnosis and correction of aniseikonia. Trans Am Acad Ophthalmol Otolaryngol 1966; 70: 340-348.

9. Gillam B. Changes in the direction of induced aniseikonic slant as a function of distance. Vision Res 1967; 7: 777-783.

10. Gillott HF. The effect on binocular vision of variations in the relative sizes and levels of illumination of the ocular images. II. Br J Phsysiol Opt 1956; 13: 218-234.
11. Achiron LR, Witkin N, Primo S, Broocker G. Contemporary management of aniseikonia. Surv Ophthalmol 1997; 41: 321-330.

12. de Wit GC. Evaluation of a new direct-comparision aniseikonia test. Binocul Vis Strabismus Q 2003; 18: 87-94.

13. Awaya S, Romano PE. Aniseikonia test: valid $=$ «true» but «true» is not equal to $p<$ or $=0.05$ or $p$ anything. The NAT is useful. Binocul Vis Strabismus Q 2003; 18: 69-71.

14. Weiss JB. Noveaux tests pour la mesure de l'anniseiconie. Bull Soc Opht France 1981; 1: 123-126.

15. Winn B, Ackerley RG, Brown CA, Murray FK, Prais J, John MF. Reduced aniseikonia in axial anisometropia with contact lens correction. Ophthalmic Physiol Opt 1988; 8: 341-344.

16. Fontaine $M$, Luca $F$, Barthelot P.Aniseikomia responsible for poor fusion? Bull Mem Soc Fr Ophtalmol 1976; 88. 191-193.

17. Gillott HF. The effect on binocular vision of the variations in the relative sizes and levels of illumination of the ocular images. III. Br J Phsysiol Opt 1957; 14: 43-58.

18. Duke-Elder S. Refracción. Teoria y práctica. Barcelona: Ed Jims; 1985; 109-114.

19. Hughes WL. Aniseikonia in emetropia. Am J Opthalmol 1937; 20: 887-890.

20. Remole A. Anisophoria and aniseikonia. Part I. The relation between optical anisophoria and aniseikonia. Optom Vis Sci 1989; 66: 659-670.

21. Bannon RE, Neumueller J, Boeder P, Burian HM. Aniseikonia and space perception--after 50 years. Am J Optom Arch Am Acad Optom 1970; 47: 423-441. 